\title{
Zheng's Scaling Laws of Jet Produced by Shaped Charge
}

\author{
Bo-Hua SUN ${ }^{1}$ \\ ${ }^{1}$ Institute of Mechanics and Technology 83 School of Civil Engineering \\ Xi'an University of Architecture and Technology, Xi'an 710055, China \\ email: sunbohua@xauat.edu.cn
}

(Dated: September 3, 2019)

\begin{abstract}
This Letter revisits the instability of jet produced by shaped charge. Scaling laws for shaped charge jet are derived by dimensional analysis. This study shows that cross-section shrinkage rate is not an independent variable and should not been included in the formulation. The universal scaling laws are formulated.
\end{abstract}

Armor-piercing bullets is a high-speed metal jet formed by a charged chargewith a metal-lined cavity inside to destroy armored targets such as tanks. The theoretical study of penetrating jet began during World War II. The most representative jet penetration theory is the incompressible fluid theory established by G.I. Taylor in the 1940s [1]. Taylor cleverly used Bernoulli's equation in incompressible fluid mechanics to establish the ratio of jet penetration depth to jet length. The concise and important relationship between them has become the main theoretical basis for the design of penetrating armor in the late World War II [2].

A large number of experiments show that during the flight of a high-speed metal jet produced by shaped charge, necking instability occurs at different location$\mathrm{s}$, and then it is pulled off. The original continuous and complete jet is broken into many small segments. The necking, especially after the breaking, the jet has severely lost its ability to penetrate the targets. Therefore, it is particularly important to study the law of necking instability and breaking of the jet, and to reveal the influencing factors of this phenomenon and the physical mechanism behind the process [2].

In the mid-1970s, Zheng (C.M. Cheng) [3, 4] realized the important engineering and theoretical value of this problem during the early research of the penetration of armor-piercing projectiles. His study shown that the instability criterion, necking and fracture time follow different laws for both low-speed and high-speed portion of the jet. At low speed, the instability of the jet is controlled by the strength (it means that jet velocity can be ignored), and the instability at the high speed section originates from the disturbance of high-speed air $[2,6 ?$ ].

For the jet instability at low-speed, Zheng (C.M. Cheng) $[3,4]$ cleverly introduced the "area impulse", namely, $\Omega=A_{0} / \dot{\varepsilon}_{0}$, to characterize the combined effect of the initial diameter of the jet and the velocity gradient (strain rate), where $A_{0}$ is initial cross-section area of jet and $\dot{\varepsilon}_{0}=\left(\frac{d \varepsilon}{d t}\right)_{0}$ is strain rate of jet material.

Through dimensional analysis, the position and time expression of jet necking and breaking is obtained by Zheng $[3,4]$, where the time $t_{\mathrm{B}}$ and diameter $d_{\mathrm{B}}$ to breakup are given by

$$
t_{\mathrm{B}}=C(\psi)\left(\Omega \frac{\rho}{Y}\right)^{1 / 3}
$$

and

$$
d_{\mathrm{B}}=D(\psi)\left(\Omega \sqrt{\frac{Y}{\rho}}\right)^{1 / 3},
$$

respectively, in which $\rho$ is jet density, $Y$ is jet yield stress, and $C(\psi)$ and $D(\psi)$ are the function of the section shrinkage $\psi$.

Zheng [3, 4] proposed a design rule for the armorpiercing ammunition-shaped cover material, that is, the material-shaped cover material must have a sufficiently high sound velocity, sufficient ductility, high density and low strength. In fact, the selection of material for armorpiercing ammunition in China is based on the above understanding [2].

Although the Zheng's scaling laws in Eqs. (1,2) have been proposed and formulated for more than 40 years, however, the functions $C(\psi)$ and $D(\psi)$ have not been determined by either computation and experiments. This is a problem that needs to be solved.

In this Letter, we show that the cross-section shrinkage $\psi$ is not a independent variable and should not be considered in the formulation. This understanding will lead a surprising result, that is, the both $C(\psi)$ and $D(\psi)$ are universal constants rather than the function of $\psi$.

For the jet produced by shaped charge with initial cross-section $A_{0}$ and the cross-section $A_{\mathrm{B}}$ at breakage, the cross-section shrinkage $\psi$ is defined as

$$
\psi=1-\frac{A_{\mathrm{B}}}{A_{0}} .
$$

Obviously, $A_{0}, A_{\mathrm{B}}$ and $\psi$ are not completely independent, because $\psi$ has a relation with $A_{0}$ and $A_{d}$ in Eq.(3). Therefore, the cross-section shrinkage $\psi$ is not a independent variable and should not be considered in the formulation when we wish to find the break-up cross-section area $A_{\mathrm{B}}$ for a given $A_{0}$.

To find the breaking area $A_{\mathrm{B}}$, the instability problem of the jet is controlled by 4 variables, such as $Y, \rho, \Omega$ and $A_{\mathrm{B}}$, whose dimensions are listed in table $\mathrm{I}$ : 
TABLE I: Dimensions of jet produced by shaped charge

\begin{tabular}{cccc}
\hline$Y$ & $\rho$ & $\Omega$ & $A_{\mathrm{B}}$ \\
\hline$L^{-1} m t^{-2}$ & $m L^{-3}$ & $L^{2} t$ & $L^{2}$ \\
\hline
\end{tabular}

The cross-section area to breakup $A_{\mathrm{B}}$ is then given by

$$
A_{\mathrm{B}}=f(\rho, Y, \Omega)
$$

which has $n=4$ variables with 3 basic dimensions $m, t, L$, one dimensionless $\Pi$ can be generated. Taking $\rho, Y$ and $\Omega$ as the basic quantities, the dimensionless $\Pi$ can be expressed as follows:

$$
\Pi=A_{\mathrm{B}} \rho^{a} Y^{b} \Omega^{c} .
$$

Since $\Pi$ is dimensionless, hence, we obtain $a=\frac{1}{3}, b=-\frac{1}{3}$ and $c=-\frac{2}{3}$.

According to dimensional theory $[5,6]$, the dimensionless format of expression of Eq. 4 is given by

$$
\Pi=f(\text { nothing }),
$$

therefore, $\Pi$ can only be a constant, namely

$$
A_{\mathrm{B}} \rho^{1 / 3} Y^{-1 / 3} \Omega^{-2 / 3}=\text { const. }
$$

hence, we have the cross-section area to breakup

$$
A_{\mathrm{B}}=\text { const. }\left(\Omega \sqrt{\frac{Y}{\rho}}\right)^{2 / 3} .
$$

Substitute Eq.8 into Eq.3, we have the cross-section shrinkage as follows

$$
\psi=1-\text { const. }\left(\frac{Y}{\rho A_{0} \dot{\varepsilon}_{0}^{2}}\right)^{1 / 3} .
$$

Assuming the jet has a circular cross-section, it leads to $A_{\mathrm{B}}=\pi d_{\mathrm{B}}^{2}$, where $d_{\mathrm{B}}$ is jet breaking diameter and can be expressed as follows

$$
d_{\mathrm{B}}=C_{\mathrm{d}}\left(\Omega \sqrt{\frac{Y}{\rho}}\right)^{1 / 3}
$$

where $C_{\mathrm{d}}$ is a constant. The illustrative plotting of the break diameter is shown in Fig.1.

Similarly, to find the time to breakup $t_{\mathrm{B}}$, the problem has 4 variables, such as $Y, \rho, \Omega$ and $t_{\mathrm{B}}$, whose dimensions are listed in table II:

TABLE II: Dimensions of jet produced by shaped charge

$$
\begin{array}{ccc}
\hline & \rho & \Omega \quad t_{\mathrm{B}} \\
\hline L^{-1} m t^{-2} & m L^{-3} & L^{2} t \quad t \\
\hline
\end{array}
$$

The breaking time $t_{\mathrm{B}}$ is then given by

$$
t_{\mathrm{B}}=g(\rho, Y, \Omega)
$$

The problem has $n=4$ variables with 3 basic dimensions $m, t, L$, one dimensionless $\Pi$ can be generated. Taking $\rho, Y$ and $\Omega$ as the basic quantities, the dimensionless $\Pi$ can be expressed as follows: $\Pi=t_{\mathrm{B}} \rho^{a} Y^{b} \Omega^{c}$. Since $\Pi$ is dimensionless, hence, we obtain $a=-\frac{1}{3}, b=\frac{1}{3}$ and $c=-\frac{1}{3}$.

According to dimensional theory $[5,6]$, the dimensionless $\Pi=f$ (nothing), therefore, $\Pi$ can only be a constant, namely $t_{\mathrm{B}} \rho^{-1 / 3} Y^{1 / 3} \Omega^{-1 / 3}=$ const., hence

$$
t_{\mathrm{B}}=C_{\mathrm{t}}\left(\Omega \frac{\rho}{Y}\right)^{1 / 3}
$$

where $C_{\mathrm{t}}$ is a constant. The illustrative plotting of the break diameter is shown in Fig.1.
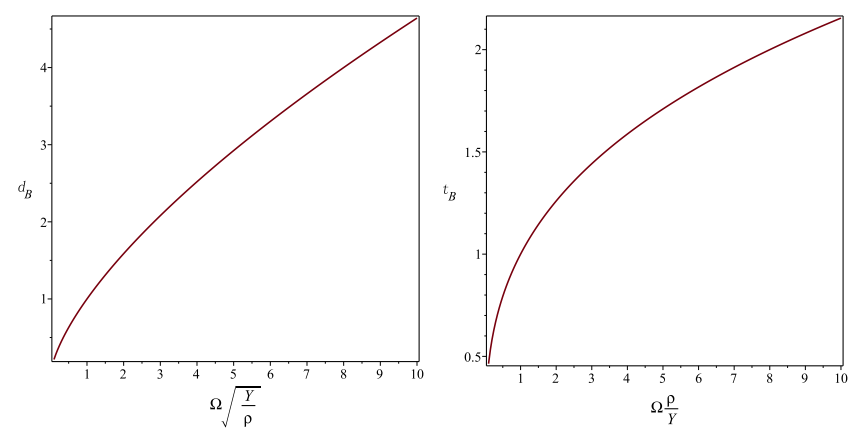

FIG. 1: The diameter $t_{\mathrm{B}}$ and time $t_{\mathrm{B}}$ to breakup.

The above formulations have shown that the crosssection functions $C(\psi)$ and $D(\psi)$ in Eqs. $(1,2)$ are constant for all jet. The constant nature of the cross-section functions $C(\psi)$ and $D(\psi)$ are of great important and makes the relations in Eq.10 and Eq.12 universal scaling laws.

In recognition of Zheng's contribution to the stability analysis of jet produced by shaped charge, the relations in Eq.10 and Eq.12 are called as Zheng's scaling laws.

[1] G.I. Taylor, A formulation of Mr. Tuck's conception of Munroe jets (1943). Scientific. Papers of G.I. Taylor, New York:Cambridge University Press. 1962, 358 - 362

[2] L.H. Dai, A pioneer in the frontier of engineering science-Zhe-Min Zheng. Advances in Mechanics, 2013, 43(3): 265-294

[3] C.M. Cheng (Z.M. Zheng), Stability of shaped charge jet. Res. Rept., Institute of Mechanics, CAS. (1977). Collected Works of Cheng C M, pp. $303-320$, Beijing: Science Press, (2004). (in Chinese)

[4] Z.M. Zheng, Stability of jet produced by shaped charge. Explosion and Shock Waves, 1: 6-17, 1981 (in Chinese)

[5] Bridgman, P.W. Dimensional Analysis. Yale University Press, New Haven (1922)

[6] Q.M. Tan, Dimensional Analysis, Springer-Verlag, Berlin Heidelberg (2011) 